\title{
Explicit formulas for the Green's function and the Bergman kernel for monogenic functions in annular shaped domains in $\mathbb{R}^{n+1}$
}

\author{
Denis Constales, Dennis Grob and Rolf Sören Kraußhar
}

\begin{abstract}
By applying a reflection principle we set up fully explicit representation formulas for the harmonic Green's function for orthogonal sectors of the annulus of the unit ball of $\mathbb{R}^{n}$. From the harmonic Green's function we then can determine the Bergman kernel function of Clifford holomorphic functions by applying an appropriate vector differentiation. As a concrete application we give an explicit analytic representation formula of the solutions to an $n$-dimensional Dirichlet problem in annular shaped domains that arises in the context of heat conduction.
\end{abstract}

Keywords: Bergman kernel, Green's function, annular domains, Clifford and Harmonic analysis, Dirichlet problems, heat conduction

AMS Subject-Classification: 30 G 35; 32 A 25, 31 B 20

\section{Introduction and Basic Notions}

Clifford analysis is the study of Dirac type operators on manifolds in $n$ real variables. Since the Dirac operator linearizes the Laplacian, its associated function theory provides us with powerful tools to study many boundary value problems from harmonic analysis in this context. In this paper we look at particular heat conduction problems in orthogonal ball sectors of the annulus of the unit ball. To apply the powerful techniques of Dirac type operators and their associated function theory to this context, we first need to introduce some basic concepts of Clifford algebras and its related analysis.

Let $\left\{e_{1}, e_{2}, \ldots, e_{n}\right\}$ be the standard basis of the Euclidean vector space $\mathbb{R}^{n}$. Further, let $C l_{0 n}$ be the associated real Clifford algebra in which

$$
e_{i} e_{j}+e_{j} e_{i}=-2 \delta_{i j} e_{0}, \quad i, j=1, \cdots, n,
$$

Financial support from BOF/GOA 01GA0405 of Ghent University gratefully acknowledged. 
holds. Here $\delta_{i j}$ is Kronecker symbol. Each element $a \in C l_{0 n}$ can be represented in the form $a=\sum_{A} a_{A} e_{A}$ with $a_{A} \in \mathbb{R}, A \subseteq\{1, \cdots, k\}, e_{A}=e_{l_{1}} e_{l_{2}} \cdots e_{l_{r}}$, where $1 \leq$ $l_{1}<\cdots<l_{r} \leq n, \quad e_{\emptyset}=e_{0}=1$. The scalar part of $a$, denoted by $\operatorname{Sc}(a)$, is defined as the $a_{0}$ term. The Clifford conjugate of $a$ is defined by $\bar{a}=\sum_{A} a_{A} \bar{e}_{A}$, where $\bar{e}_{A}=$ $\bar{e}_{l_{r}} \bar{e}_{l_{r-1}} \cdots \bar{e}_{l_{1}}$ and $\bar{e}_{j}=-e_{j}$ for $j=1, \cdots, n, \bar{e}_{0}=e_{0}=1$. For the space of paravectors $\mathbb{R} \oplus \mathbb{R}^{n} \subset C l_{0 n}$, which consists of elements of the form $z=z_{0}+z_{1} e_{1}+z_{2} e_{2}+\cdots+z_{n} e_{n}$, we also write $\mathbb{R}^{n+1}$ for simplicity. The standard scalar product between two Clifford numbers $a, b \in C l_{0 n}$ is defined by $\langle a, b\rangle:=\operatorname{Sc}(a \bar{b})$. This induces a pseudo norm on the Clifford algebra, viz $\|a\|=\left(\sum_{A}\left|a_{A}\right|^{2}\right)^{1 / 2}$.

The simplest case of a manifold in $n+1$ real variables is of course the $n+1$-dimensional Euclidean paravector space $\mathbb{R}^{n+1}$ with the flat metric. All the basic concepts that are going to be introduced next can be adapted to more general classes of Riemannian manifolds, see for instance $[5,16]$ and elsewhere. It is just for simplicity that we take $\mathbb{R}^{n+1}$ as base manifold in this paper. The results that we develop for the concrete heat conductions problems can be adapted to the context of considering appropriate analogies of these PDE in the context of other classes of Riemannian spin manifolds. This will be done in a follow up paper.

In the Euclidean flat space $\mathbb{R}^{n+1}$, the Dirac operator has the simple form

$$
D_{z}=\frac{\partial}{\partial z_{0}}+\sum_{j=1}^{n} \frac{\partial}{\partial z_{j}} e_{j} \quad z_{j} \in \mathbb{R} j=0, \ldots, n .
$$

In this particular context it is often called the generalized Cauchy-Riemann operator. Next let $\Omega \subseteq \mathbb{R}^{n+1}$ be an open set. A real differentiable function $f: \Omega \rightarrow C l_{0 n}$ that satisfies inside of $\Omega$ the system $D_{z} f=0$ (or $f D_{z}=0$ ), is called left (right) monogenic with respect to the paravector variable $z$, respectively. In the two-dimensional case $(n=1)$ this operator coincides with the classical complex Cauchy-Riemann operator. In this sense, the set of monogenic functions can also be regarded as a higher dimensional generalization of the set of complex-analytic functions. For details, see for example [15] and elsewhere. The linear operator $D_{z}$ factorizes the Euclidean Laplacian, viz $D_{z} \bar{D}_{z}=\Delta_{z}$. Each real component of a monogenic function is therefore Euclidean harmonic. This allows us to apply methods from harmonic analysis to study monogenic functions but also vice versa. Notice that also in the framework of other Riemannian manifolds, the Dirac operator has the property that it linearizes the Laplacian. It is thus a powerful object to do harmonic analysis in a very global way.

In particular, the study of related Hilbert spaces leads to important applications to boundary value problems from harmonic analysis that appear in many branches of physics and the applied sciences, as for instance in the treatment of the Navier-Stokes system with heat conduction, see for instance [17] and our recent paper [12].

An important example of a Hilbert space of Clifford valued monogenic functions that satisfies in particular the Bergman condition $\|f(z)\| \leq C(z)\|f\|_{L^{2}}$ is the space of square integrable monogenic functions in a domain of $\mathbb{R}^{n+1}$. This is often called the Bergman space of monogenic functions. Each Bergman space is a Banach space which 
is endowed with the Clifford-valued inner product $(f, g)=\int_{\Omega} \overline{f(z)} g(z) d V$, where $f$ and $g$ denote left monogenic functions that are square integrable on a given domain $\Omega \subseteq \mathbb{R}^{n+1}$.

Some of the first contributions to the investigation of hypercomplex function spaces came from R. Delanghe and F. Brackx in 1976 and 1978, see [2, 14], and then later on in $[1,6,7,8,20,21,22]$, among a lot of others.

The reproducing kernel of the Bergman space, called the Bergman kernel, is uniquely defined and satisfies $f(z)=\int_{\Omega} B_{\Omega}(z, w) f(w) d V$ for any square integrable monogenic function $f$ on the domain $\Omega \subseteq \mathbb{R}^{n+1}$.

A central topic is the determination of explicit and closed formulas for these kernel functions. This is very difficult in general. In contrast to the Cauchy kernel, the Bergman kernel depends on the domain $\Omega$.

For instance, in [2, 6], one finds explicit formulas for the monogenic Bergman kernel for the unit ball and the half-space. Explicit formulas for the monogenic Bergman kernels for rectangular and strip domains have been worked out recently in [9]. The Bergman kernel appears as an infinite sum that is composed of translation mirror images of the Cauchy-Riemann derivative of the monogenic Cauchy kernel.

In [10] explicit formulas for the Bergman kernels of wedge shaped domains have been developed.

In [5] D. Calderbank gave a representation formula for the Szegö kernel in full annulus of the unit ball. The Szegö kernel is the analogue of the Bergman kernel, when passing from volume integrals to surface integrals.

The main goal of this paper is to extend D. Calderbank's result from [5] and to develop a fully explicit formula for the reproducing Bergman kernel for arbitrary orthogonal sectors of the annulus of the $(n+1)$-dimensional unit ball in the framework of the generalized Cauchy-Riemann operator.

Our method bases on first determining an explicit formula for the harmonic Green's function of this family of domains in terms of an infinite series over the classical Green's kernel of $\mathbb{R}^{n+1}$ summed over a discrete dilatation group. This arises from successively adding up correction terms corresponding to reflections (or inversions) across the different boundaries of the domain. From the Green's function we then can determine the Bergman kernel function by applying from the right a CauchyRiemann operator and from the left its conjugate in the other variable.

The annulus of the half-ball and the annulus of the full unit ball are included as special cases. For clarity we start with the Green's function of the full annulus, giving a detailed proof for it, and show next how the technique developed previously for wedge shaped domains in [10] can be applied to extend the results to the annulus of the half-ball and, even more generally, to annuli of arbitrary orthogonal ball sectors.

Finally we discuss as a concrete application an explicit analytic representation formula of the solutions to the Dirichlet problem in annular shaped domains that arise for instance in the context of heat conduction. In turn it also provides an application of the Clifford algebra valued operator calculus developed in [17] in terms of explicitness. 


\section{The full annulus of the unit ball}

Throughout the whole paper let $\lambda$ be a fixed real number in the open interval $] 0,1[$. Here and in all that follows we denote by

$$
B(0, \lambda, 1):=\left\{z \in \mathbb{R}^{n+1}|\lambda<| z \mid<1\right\}
$$

the full annulus with radii $\lambda$ and 1 .

In [5] D. Calderbank gave an explicit formula for the Szegö kernel of the annulus $B(0, \lambda, 1)$, but without giving a detailed proof. In this section we start with giving the formula for the Green's kernel and provide a detailed proof, because we need this result and the technique of the proof later in all that follows.

Theorem 1. (compare with [5])

The function

$$
\begin{aligned}
\mathcal{G}(z, w) & :=-\frac{1}{(n-1) A_{n+1}}\left\{\frac{1}{|1-\bar{z} w|^{n-1}}+\sum_{k \in \mathbb{Z}}{ }^{\prime}\left[\frac{\lambda^{k(n-1)}}{\left|1-\lambda^{2 k} \bar{z} w\right|^{n-1}}-\frac{\lambda^{k(n-1)}}{\left|w-\lambda^{2 k} z\right|^{n-1}}\right]\right\} \\
& =-\frac{1}{(n-1) A_{n+1}}\left\{\frac{1}{|w-z|^{n-1}}+\sum_{k \in \mathbb{Z}}\left[\frac{\lambda^{k(n-1)}}{\left|1-\lambda^{2 k} \bar{z} w\right|^{n-1}}-\frac{\lambda^{k(n-1)}}{\left|w-\lambda^{2 k} z\right|^{n-1}}\right]\right\}
\end{aligned}
$$

is the harmonic Green's function of the annulus $B(0, \lambda, 1)$. Here and in all that follows, the prime behind the summation sign means that the term associated to the index $k=0$ is omitted in the summation.

Remark. Notice that both expressions are clearly equivalent; the first one offers the advantage of showing that the function has no singularities inside the annulus, the second will lead to a shorter proof involving a telescopic series.

Proof. The main deal is to show that the series inside the expression $\mathcal{G}(z, w)$ converges for each point $w$ of the boundary to the expression $\frac{1}{|w-z|^{n-1}}$. Notice that the boundary of the annulus of the full unit ball consists of two parts; one sphere of radius $\lambda$ and another sphere of radius 1.

1. Case: Let $w$ be a boundary point of the annulus with $|w|=1$.

Each point of this boundary part satisfies

$$
\frac{\lambda^{k(n-1)}}{\left|1-\lambda^{2 k} \bar{z} w\right|^{n-1}} \stackrel{|\bar{w}|=1}{=} \frac{\lambda^{k(n-1)}}{\left|1-\lambda^{2 k} \bar{z} w\right|^{n-1}|\bar{w}|^{n-1}}=\frac{\lambda^{k(n-1)}}{\left|\bar{w}-\lambda^{2 k} \bar{z}\right|^{n-1}}=\frac{\lambda^{k(n-1)}}{\left|w-\lambda^{2 k} z\right|^{n-1}}
$$

and the series is identically zero. Then also

$$
\frac{1}{|1-\bar{z} w|^{n-1}}=\frac{1}{|1-\bar{z} w|^{n-1}|\bar{w}|^{n-1}}=\frac{1}{|z-w|^{n-1}} .
$$

2. Case: Now let $w$ be a boundary point of the other boundary part of the annulus satisfying $|w|=\lambda$. Each point of this boundary part satisfies

$$
\frac{\lambda^{k(n-1)}}{\left|1-\lambda^{2 k} \bar{z} w\right|^{n-1}} \stackrel{|w|=\lambda}{=} \frac{\lambda^{k(n-1)} \lambda^{n-1}}{\left|1-\lambda^{2 k} \bar{z} w\right|^{n-1}|w|^{n-1}}=\frac{\lambda^{(k+1)(n-1)}}{\left|w-\lambda^{2(k+1)} z\right|^{n-1}}
$$


so that the series telescopes. To see this, let $m \in \mathbb{N}$ be an arbitrary positive integer. Then we have

$$
\begin{aligned}
& =\sum_{|k| \leq m}\left[\frac{\lambda^{k(n-1)}}{\left|1-\lambda^{2 k} \bar{z} w\right|^{n-1}}-\frac{\lambda^{k(n-1)}}{\left|w-\lambda^{2 k} z\right|^{n-1}}\right] \\
& =\sum_{|k| \leq m}\left[\frac{\lambda^{(k+1)(n-1)}}{\left|w-\lambda^{2(k+1)} z\right|^{n-1}}-\frac{\lambda^{k(n-1)}}{\left|w-\lambda^{2 k} z\right|^{n-1}}\right] \\
& =\frac{\lambda^{(m+1)(n-1)}}{\left|w-\lambda^{2(m+1)} z\right|^{n-1}}-\frac{\lambda^{-m(n-1)}}{\left|w-\lambda^{-2 m} z\right|^{n-1}} .
\end{aligned}
$$

The first term clearly tends to 0 as $m \rightarrow+\infty$ because the numerator tends to 0 in view of $0<\lambda<1$, and $w-\lambda^{2(m+1)} z$ in the denominator tends to $w$, whose norm is positive.

To be precise, since the sequence $\left(\lambda^{(m+1)(n-1)}\right)_{m \in \mathbb{N}}$ converges to zero, the expression

$$
\left|w-\lambda^{2(m+1)} z\right|^{n-1}
$$

remains bounded for all $m \in \mathbb{N}$. Therefore, one can find a real constant $C$ such that

$$
\frac{\lambda^{(m+1)(n-1)}}{\left|w-\lambda^{2(m+1)} z\right|^{n-1}} \leq C \lambda^{(m+1)(n-1)},
$$

whose right hand-side tends to zero when $m$ tends to $+\infty$.

Multiplying the numerator and denominator by $\lambda^{2 m(n-1)}$, the second term can be rewritten

$$
\frac{\lambda^{-m(n-1)}}{\left|w-\lambda^{-2 m} z\right|^{n-1}}=\frac{\lambda^{m(n-1)}}{\left|z-\lambda^{2 m} w\right|^{n-1}}
$$

which is quite similar to the first term (replace $m$ by $m+1$, switch $z$ and $w$ ) and converges to 0 as $m \rightarrow \infty$ for the same reasons. We thus obtain the normal convergence of the whole series, majorized by a geometric series in $\lambda$.

We thus may conclude that in particular $\mathcal{G}(z, w)$ converges for any point $w$ belonging to one of the boundary parts to

$$
-\frac{1}{(n-1) A_{n+1}} \frac{1}{|w-z|^{n-1}} \text {. }
$$

Since every term in the series is harmonic in both variables $z$ and $w$, it follows in view of the normal convergence by Weierstraß' convergence theorem that the whole expression $\mathcal{G}(z, w)$ is harmonic in both variables inside the annulus.

From the Green's function we can obtain the hypercomplex Bergman kernel of monogenic functions, namely by applying from the left and the right hand side the hypercomplex differential operators $\bar{D}_{z}$ and $D_{w}$, respectively, and applying the Clifford algebra calculus. More precisely: 
Theorem 2. Let $0<\lambda<1$. Then the reproducing Bergman kernel of the full annulus with radii $\lambda$ and 1 is given by

$$
B(z, w):=-\frac{1}{(n-1) A_{n+1}} \sum_{k \in \mathbb{Z}} \bar{D}_{z} \frac{\lambda^{k(n-1)}}{\left|1-\lambda^{2 k} \bar{z} w\right|^{n-1}} D_{w} .
$$

Proof. In Theorem 1 we determined the Green's function of the full annulus. Following for example $[22,21]$ the associated Bergman kernel can be expressed as

$$
B(z, w)=\bar{D}_{z} \mathcal{G}(z, w) D_{w}
$$

The series (2) is absolutely locally uniformly convergent, as shown in detail in the proof of Theorem 1. Applying Weierstraß' convergence theorem, cf. e.g. [15], thus allows us to put the differential operators within the sum. Since the second summand in the sum in the series representation formula for the Green's kernel, i.e.

$$
\frac{\lambda^{k(n-1)}}{\left|w-\lambda^{2 k} z\right|^{n-1}}
$$

is a harmonic function of a linear combination of $w$ and $z, D_{w}$ is proportional to $D_{z}$ and therefore $\overline{D_{z}} D_{w}$ is proportional to $\overline{D_{z}} D_{z}=\Delta_{z}$,

$$
\bar{D}_{z}\left[\frac{\lambda^{k(n-1)}}{\left|w-\lambda^{2 k} z\right|^{n-1}}\right] D_{w}=0 .
$$

Hence, only the sum written down in the formula of (2) remains.

Remark: In the limit case $\lambda \rightarrow 0^{+}$one obtains the usual representation formula of the Bergman kernel for the full unit ball. In this case the whole series reduces to the single term $\bar{D}_{z} \frac{1}{|1-\bar{z} w|^{n-1}} D_{w}$ which is the well-known expression for the Bergman kernel of the full unit ball, cf. e.g. [3].

\section{The annulus of the half-ball}

The simplest non-trivial example of an orthogonal ball sector is the half-ball. In [10] we determined the reproducing Bergman kernel of the complete unit half-ball

$$
H B(0,1):=\left\{z \in \mathbb{R}^{n+1}|0<| z \mid<1, z_{0}>0\right\} .
$$

Now we want to set up an explicit representation formula for the Bergman kernel of the annulus of the half-ball, again with radii $r=\lambda \in(0,1)$ and $R=1$. This is the set

$$
H B(0, \lambda, 1):=\left\{z \in \mathbb{R}^{n+1}|\lambda<| z \mid<1, z_{0}>0\right\} .
$$

Adapting the method from the proof of Theorem 1 allows us to establish:

Theorem 3. The function

$$
\begin{array}{r}
\mathcal{G}(z, w):=-\frac{1}{(n-1) A_{n+1}}\left[\frac{1}{|w-z|^{n-1}}+\sum_{k \in \mathbb{Z}}\left[\frac{\lambda^{k(n-1)}}{\left|\bar{w}+\lambda^{2 k} z\right|^{n-1}}+\frac{\lambda^{k(n-1)}}{\left|1-\lambda^{2 k} z \bar{w}\right|^{n-1}}\right.\right. \\
\left.\left.-\frac{\lambda^{k(n-1)}}{\left|w-\lambda^{2 k} z\right|^{n-1}}-\frac{\lambda^{k(n-1)}}{\left|1+\lambda^{2 k} z w\right|^{n-1}}\right]\right]
\end{array}
$$


is the Green's function of the above described half-ball annulus $H B(0, \lambda, 1)$.

Remark: Note that the singular term before the series cancels with an identical one in the $k=0$ term, so that the expression has no singularities inside the annulus.

Proof. Again, the main deal is to show that the expression $\mathcal{G}(z, w)$ converges to $\frac{1}{|w-z|^{n-1}}$ for each boundary point of the above described half-ball annulus $H B(0, \lambda, 1)$. Notice that the boundary of $H B(0, \lambda, 1)$ is composed by four parts: Two half-spheres of radii $\lambda$ and 1 and two rectangular surfaces lying in the hyperplane $w_{0}=0$. Actually, the two boundary parts belonging to the hyperplane $w_{0}=0$ can be treated together as one case.

1.Case: Let $w$ be an element of the boundary of $H B(0, \lambda, 1)$ that satisfies $|w|=1$. Such a boundary point satisfies

$$
\left|1-\lambda^{2 k} z \bar{w}\right|^{n-1}=\left|1-\lambda^{2 k} z \bar{w}\right|^{n-1}|w|^{n-1} \stackrel{|w|=1}{=}\left|w-\lambda^{2 k} z\right|^{n-1}
$$

and analogously

$$
\left|1+\lambda^{2 k} z w\right|^{n-1} \stackrel{|w|=1}{=}\left|w^{-1}+\lambda^{2 k} z w w^{-1}\right|^{n-1}|w|^{n-1} \stackrel{w^{-1}=\bar{w}}{=}\left|\bar{w}+\lambda^{2 k} z\right|^{n-1} .
$$

Therefore, we obtain

$$
\begin{aligned}
& \frac{\lambda^{k(n-1)}}{\left|\bar{w}+\lambda^{2 k} z\right|^{n-1}}+\frac{\lambda^{k(n-1)}}{\left|1-\lambda^{2 k} z \bar{w}\right|^{n-1}}-\frac{\lambda^{k(n-1)}}{\left|w-\lambda^{2 k} z\right|^{n-1}}-\frac{\lambda^{k(n-1)}}{\left|1+\lambda^{2 k} z w\right|^{n-1}} \\
= & \frac{\lambda^{k(n-1)}}{\left|\bar{w}+\lambda^{2 k} z\right|^{n-1}}+\frac{\lambda^{k(n-1)}}{\left|w-\lambda^{2 k} z\right|^{n-1}}-\frac{\lambda^{k(n-1)}}{\left|w-\lambda^{2 k} z\right|^{n-1}}-\frac{\lambda^{k(n-1)}}{\left|\bar{w}+\lambda^{2 k} z\right|^{n-1}} \\
= & 0,
\end{aligned}
$$

so, all these terms cancel out each other. Thus, the series reduces to zero for all elements of the boundary part with $|w|=1$, so that we end with

$$
\mathcal{G}(z, w)=\frac{1}{-(n-1) A_{n+1}} \frac{1}{|w-z|^{n-1}} .
$$

2.Case: Let $w$ be a boundary point of $\operatorname{HB}(0, \lambda, 1)$ that satisfies $w_{0}=0$

Since $S c(w)=0$ all these boundary points satisfy

$$
\bar{w}=-w .
$$

Applying this rule in the expression of the sum, then we obtain

$$
\begin{aligned}
& \frac{\lambda^{k(n-1)}}{\left|\bar{w}+\lambda^{2 k} z\right|^{n-1}}+\frac{\lambda^{k(n-1)}}{\left|1-\lambda^{2 k} z \bar{w}\right|^{n-1}}-\frac{\lambda^{k(n-1)}}{\left|w-\lambda^{2 k} z\right|^{n-1}}-\frac{\lambda^{k(n-1)}}{\left|1+\lambda^{2 k} z w\right|^{n-1}} \\
= & \frac{\lambda^{k(n-1)}}{\left|w-\lambda^{2 k} z\right|^{n-1}}+\frac{\lambda^{k(n-1)}}{\left|1+\lambda^{2 k} z w\right|^{n-1}}-\frac{\lambda^{k(n-1)}}{\left|w-\lambda^{2 k} z\right|^{n-1}}-\frac{\lambda^{k(n-1)}}{\left|1+\lambda^{2 k} z w\right|^{n-1}} \\
= & 0 .
\end{aligned}
$$

So, for all elements $w$ belonging to that part of the boundary we again obtain that $\mathcal{G}(z, w)$ reduces to the expression $-\frac{1}{(n-1) A_{n+1}} \frac{1}{|z-w|^{n-1}}$. 
3. Case: Finally, let $w$ be a point of the boundary of $H B(0, \lambda, 1)$ that satisfies $|w|=\lambda$. In this case the series telescopes again. Analogously as shown in the previous section we can show that

$$
\frac{\lambda^{k(n-1)}}{\left|1-\lambda^{2 k} z \bar{w}\right|^{n-1}}=\frac{\lambda^{k(n-1)}}{\left|w-\lambda^{2 k+2} z\right|^{n-1}}|w|^{n-1}=\frac{\lambda^{(k+1)(n-1)}}{\left|w-\lambda^{2 k+2} z\right|^{n-1}} .
$$

By replacing $z$ by $-z$ and $\bar{w}$ by $w$ in this formula we also obtain the following rule:

$$
\frac{\lambda^{k(n-1)}}{\left|1+\lambda^{2 k} z w\right|^{n-1}}=\frac{\lambda^{(k+1)(n-1)}}{\left|\bar{w}+\lambda^{2 k+2} z\right|^{n-1}} .
$$

Let now $m \in \mathbb{N}$ be a fixed number. For the finite partial sum we then have

$$
\begin{aligned}
& \sum_{|k| \leq m}\left[\frac{\lambda^{k(n-1)}}{\left|\bar{w}+\lambda^{2 k} z\right|^{n-1}}+\frac{\lambda^{k(n-1)}}{\left|1-\lambda^{2 k} z \bar{w}\right|^{n-1}}-\frac{\lambda^{k(n-1)}}{\left|w-\lambda^{2 k} z\right|^{n-1}}-\frac{\lambda^{k(n-1)}}{\left|1+\lambda^{2 k} z w\right|^{n-1}}\right] \\
= & \sum_{|k| \leq m}\left[\frac{\lambda^{k(n-1)}}{\left|\bar{w}+\lambda^{2 k} z\right|^{n-1}}+\frac{\lambda^{(k+1)(n-1)}}{\left|w-\lambda^{2 k+2} z\right|^{n-1}}-\frac{\lambda^{k(n-1)}}{\left|w-\lambda^{2 k} z\right|^{n-1}}-\frac{\lambda^{(k+1)(n-1)}}{\left|\bar{w}+\lambda^{2 k+2} z\right|^{n-1}}\right] \\
= & \frac{\lambda^{-m(n-1)}}{\left|\bar{w}+\lambda^{-2 m} z\right|^{n-1}}+\frac{\lambda^{(m+1)(n-1)}}{\left|w-\lambda^{2(m+1)} z\right|^{n-1}}-\frac{\lambda^{-m(n-1)}}{\left|w-\lambda^{-2 m} z\right|^{n-1}}-\frac{\lambda^{(m+1)(n-1)}}{\left|\bar{w}+\lambda^{2(m+1) z}\right|^{n-1}}
\end{aligned}
$$

The second and the fourth term tend to zero as $m$ tends to $+\infty$, because the numerator tends to zero in view of $\lambda \in] 0,1\left[\right.$ and the expression $w-\lambda^{2(m+1)} z$ and $\bar{w}+\lambda^{2(m+1)} z$ in the denominator tend to $w$ resp. to $\bar{w}$, whose norm is non-zero. Multiplying the numerator and denominator of the first and the third term by $\lambda^{2 m(n-1)}$, then the resp. expressions can be rewritten as

$$
\frac{\lambda^{-m(n-1)}}{\left|\bar{w}+\lambda^{-2 m} z\right|^{n-1}}=\frac{\lambda^{m(n-1)}}{\left|z+\lambda^{2 m} \bar{w}\right|^{n-1}}
$$

resp.

$$
\frac{\lambda^{-m(n-1)}}{\left|w-\lambda^{-2 m} z\right|^{n-1}}=\frac{\lambda^{m(n-1)}}{\left|z-\lambda^{2 m} w\right|^{n-1}} .
$$

These terms again converge to zero as $m$ tends to $+\infty$. With the same arguments as applied in the proof of Theorem 1 we can conclude that the series is normally convergent, again using geometric series in $\lambda$ as convergent majorants.

With similar arguments as applied to Theorem 2 we can now establish

Theorem 4. Let $0<\lambda<1$. Then the Bergman kernel of the annulus of the half-ball is given by

$$
\begin{aligned}
\mathcal{B}(z, w):=-\frac{1}{(n-1) A_{n+1}}\left[\sum _ { k \in \mathbb { Z } } \overline { D } _ { z } \left[\frac{\lambda^{k(n-1)}}{\left|\bar{w}+\lambda^{2 k} z\right|^{n-1}}+\frac{\lambda^{k(n-1)}}{\left|1-\lambda^{2 k} z \bar{w}\right|^{n-1}}\right.\right. \\
\left.\left.-\frac{\lambda^{k(n-1)}}{\left|1+\lambda^{2 k} z w\right|^{n-1}}\right] D_{w}\right] .
\end{aligned}
$$


Proof. Again we obtain the Bergman kernel by applying the formula

$$
B(z, w)=\bar{D}_{z} \mathcal{G}(z, w) D_{w}
$$

to the series representation of Theorem 3. Since the series is normally convergent we can put the operators $\bar{D}_{z}$ and $D_{w}$ inside the series expression.

Again all terms of the form

$$
\bar{D}_{z} \frac{1}{\left|w-\lambda^{2 k} z\right|^{n-1}} D_{w}
$$

vanish for all $k \in \mathbb{Z}$ for the reason mentioned in the proof of Theorem 2. All the other terms, however, remain.

In the case where $\lambda \rightarrow 0^{+}$the expression clearly reduces to

$$
\bar{D}_{z}\left(\frac{1}{|z+\bar{w}|^{n-1}}+\frac{1}{|1-z \bar{w}|^{n-1}}-\frac{1}{|1+z w|^{n-1}}\right) D_{w}
$$

This is the representation of the unit half-ball that we worked out previously in [10].

\section{Annuli of orthogonal ball sectors}

In this section we present our main result. This one includes the previous two formulas as particular subcases.

The half-ball, quarter-ball and the eighth-ball are the simplest examples that belong to the family of orthogonal ball sectors. An orthogonal ball sector of the unit ball in $\mathbb{R}^{n+1}$, denoted by $\mathcal{S}_{0,1}$, is defined by the following inequalities:

$$
\mathcal{S}_{0,1 ; k_{1}}:=\left\{z \in \mathbb{R}^{n+1}\left|z_{0}>0, \ldots, z_{k_{1}-1}>0, \quad\right| z \mid<1\right\},
$$

where $k_{1}$ is the number of flat surfaces in its boundary, $1 \leq k_{1} \leq n+1$.

Let us now consider annuli of these orthogonal ball sectors with radii $\lambda$ and 1 where again $0<\lambda<1$. Precisely speaking, these have the form

$$
\mathcal{S}_{0, \lambda, 1 ; k_{1}}:=\left\{z \in \mathbb{R}^{n+1}\left|z_{0}>0, \ldots, z_{k_{1}-1}>0, \quad \lambda<\right| z \mid<1\right\},
$$

where again $k_{1}$ is the number of flat surfaces in the boundary, $1 \leq k_{1} \leq n+1$.

We write $K_{1}=\left\{0,1,2, \ldots, k_{1}-1\right\}$. Furthermore, for any paravector $w$ and subset $A \subseteq\{0, \ldots, n\}$ we denote by $w^{A}$ the paravector obtained by changing the sign of all components whose index is an element of $A$, i.e.,

$$
w^{A}=\sum_{j \notin A} w_{j} e_{j}-\sum_{j \in A} w_{j} e_{j} .
$$

With these notations we can formulate our main theorem

Theorem 5. The function

$$
-\frac{1}{(n-1) A_{n+1}}\left\{\frac{1}{|w-z|^{n-1}}+\sum_{k \in \mathbb{Z}} \sum_{A \subseteq K_{1}}(-1)^{|A|}\left[\frac{\lambda^{k(n-1)}}{\left|1-\lambda^{2 k} z \bar{w}^{A}\right|^{n-1}}-\frac{\lambda^{k(n-1)}}{\left|w^{A}-\lambda^{2 k} z\right|^{n-1}}\right]\right\}
$$

is the harmonic Green's function of the annulus of the orthogonal ball sector $\mathcal{S}_{0, \lambda, 1, k_{1}}$. 
Proof. First we note that for all subsets $A \subseteq K_{1}$ there holds

$$
\left|w^{A}\right|=|w| \text {. }
$$

This allows us to establish completely analogously to the case of the annulus of the full unit ball that the series

$$
\sum_{k \in \mathbb{Z}}(-1)^{|A|}\left[\frac{\lambda^{k(n-1)}}{\left|1-\lambda^{2 k} z \bar{w}^{A}\right|^{n-1}}-\frac{\lambda^{k(n-1)}}{\left|w^{A}-\lambda^{2 k} z\right|^{n-1}}\right]
$$

converges absolutely locally uniformly for any fixed $A \subseteq K_{1}$. Since $K_{1}$ is a finite set, the inner sum is a finite sum. Therefore, the whole series converges absolutely locally uniformly. Let us now consider its behavior at the boundary points.

1.Case: $|w|=1$.

In this case we, in view of $\left|w^{A}\right|=|w|$, can use the same method as in the first case of Theorem 4 , and conclude that for the boundary points $w$ each pair of terms appearing in the sum thus cancels out for each single $k \in \mathbb{Z}$ and each $A \subseteq K_{1}$ so that only the term

$$
\frac{1}{(1-n) A_{n+1}} \frac{1}{|z-w|^{n-1}}
$$

remains.

2.Case: $|w|=\lambda$.

Let $m \in \mathbb{N}$ be a fixed integer.

Again, relying on $\left|w^{A}\right|=|w|$, for all these boundary points we obtain

$$
\begin{aligned}
& \sum_{|k| \leq m} \sum_{A \subseteq K_{1}}(-1)^{|A|}\left[\frac{\lambda^{(k+1)(n-1)}}{\left|w^{A}-\lambda^{2(k+1)} z\right|^{n-1}}-\frac{\lambda^{k(n-1)}}{\left|w^{A}-\lambda^{2 k} z\right|^{n-1}}\right] \\
= & \sum_{A \subseteq K_{1}}(-1)^{|A|}\left[\frac{\lambda^{(m+1)(n-1)}}{\left|w^{A}-\lambda^{2(m+1)} z\right|^{n-1}}-\frac{\lambda^{-m(n-1)}}{\left|w^{A}-\lambda^{-2 m}\right|^{n-1}}\right],
\end{aligned}
$$

by applying the same arguments as in the case of the half-ball.

Analogously to the proof of Theorem 3 we can then conclude that each term of this finite sum tends to zero when $m$ tends to $+\infty$, so the whole series vanishes identically.

3.Case: Let $w$ be a boundary point that satisfies $w_{j}=0$ for an arbitrary but fixed $j \in K_{1}$.

Since the $j$-th component of all these points $w$ vanish, we can write instead of $w^{A}$ equivalently $w^{A \triangle\{j\}}, A \subseteq K_{1}$. This allows us to rewrite

$\frac{(-1)^{|A|+1} \lambda^{k(n-1)}}{\left|w^{A}-\lambda^{2 k} z\right|^{n-1}}=\frac{(-1)^{|A|+1} \lambda^{k(n-1)}}{\left|w^{A \triangle\{j\}}-\lambda^{2 k} z\right|^{n-1}}=\frac{-(-1)^{|A|+2} \lambda^{k(n-1)}}{\left|w^{A \triangle\{j\}}-\lambda^{2 k} z\right|^{n-1}}=\frac{-(-1)^{|A \triangle\{j\}|+1} \lambda^{k(n-1)}}{\left|w^{A \triangle\{j\}}-\lambda^{2 k} z\right|^{n-1}}$

For $A \triangle\{j\}$ we thus obtain the same expression as for $A$ but with the opposite sign. Since the summation extends over all subsets $A \subseteq K_{1}$, for each $A \subseteq K_{1}$ the corresponding term for $A \triangle\{j\}$ with opposite sign is always included in the sum. 
Analogously we can show that

$$
\frac{(-1)^{|A|+1} \lambda^{k(n-1)}}{\left|1-\lambda^{2 k} z \bar{w}^{A}\right|^{n-1}}=\frac{-(-1)^{|A \triangle\{j\}|+1} \lambda^{k(n-1)}}{\left|1-\lambda^{2 k} z \bar{w}^{A \triangle\{j\}}\right|^{n-1}} .
$$

Thus, the whole inner sum vanishes for each $k \in \mathbb{Z}$. Since the series converges absolutely, the whole expression for $\mathcal{G}(z, w)$ thus reduces to the single term

$$
\frac{1}{(1-n) A_{n+1}} \frac{1}{|z-w|^{n-1}}
$$

for all these boundary points. Clearly, each expression in the sum is harmonic in $z$ and $w$. In view of the normal convergence of the sum we may conclude that $\mathcal{G}(z, w)$ is the harmonic Green's function of the annulus part of the orthogonal ball sector.

Finally we can establish our main theorem

Theorem 6. The function

$$
\begin{aligned}
& B(z, w)=-\frac{1}{(n-1) A_{n+1}}\left\{\sum_{k \in \mathbb{Z}}\left[\bar{D}_{z} \frac{\lambda^{k(n-1)}}{\left|1-\lambda^{2 k} z \bar{w}\right|^{n-1}} D_{w}\right]\right\} \\
&-\frac{1}{(n-1) A_{n+1}}\left\{\sum _ { k \in \mathbb { Z } \emptyset \neq A \subseteq K _ { 1 } } ( - 1 ) ^ { | A | } \left[\bar{D}_{z} \frac{\lambda^{k(n-1)}}{\left|1-\lambda^{2 k} z \bar{w}^{A}\right|^{n-1}} D_{w}\right.\right. \\
&\left.\left.-\bar{D}_{z} \frac{\lambda^{k(n-1)}}{\left|w^{A}-\lambda^{2 k} z\right|^{n-1}} D_{w}\right]\right\}
\end{aligned}
$$

is the monogenic Bergman kernel of the annulus of the orthogonal ball sector $\mathcal{S}_{0, \lambda, 1 ; k_{1}}$.

Proof. Again, we obtain the Bergman kernel from the representation of the Green's function by applying the formula

$$
B(z, w)=\bar{D}_{z} \mathcal{G}(z, w) D_{w} .
$$

Again, the series representation in Theorem 5 is normally convergent which allows us to put the differential operators inside the sum. Notice that only the terms of the form $\frac{1}{\left|w-\lambda^{2 k} z\right|^{n-1}}$ are harmonic functions of a linear combination of $w$ and $z$. Only these are canceled out identically when applying the operator $\bar{D}_{z}$ from the left and $D_{w}$ from the right. Notice that this particular situation only appears in the cases where $A=\emptyset$. So, most of the terms remain.

\section{Remarks:}

- When considering $\lambda \rightarrow 0^{+}$we obtain the representation formulas for the complete orthogonal ball sectors, that were worked out in [10].

- In the particular setting of three dimensions, where we are in $\mathbb{R}^{2+1}$, the only orthogonal ball sectors are half-balls, quarter-balls and eighth-balls. Remember that the number of flat surfaces $k_{1}$ has to satisfy $k_{1} \leq n+1$, so $k_{1} \leq 3$ in the three dimensional case. In the simplest case where $K_{1}=\{0\}$ which corresponds to $k_{1}=1$ we are dealing with the half-ball treated in the previous section. 
- It is easy to adapt the formulas for the case of annuli of orthogonal ball sectors of general radii $0<r<R<+\infty$. By a simple re-scaling argument we directly obtain that the Green's function of the annulus with radii $r$ and $R$ has the form

$$
\begin{aligned}
\mathcal{G}(z, w)=- & \frac{1}{(n-1) A_{n+1}}\left\{\frac{1}{|w-z|^{n-1}}\right. \\
& \left.+\sum_{k \in \mathbb{Z}}\left[\frac{\left(\frac{r}{R}\right)^{k(n-1)}}{\left|R-\left(\frac{r}{R}\right)^{2 k} \frac{\bar{z} w}{R}\right|^{n-1}}-\frac{\left(\frac{r}{R}\right)^{k(n-1)}}{\left|w-\left(\frac{r}{R}\right)^{2 k} z\right|^{n-1}}\right]\right\} .
\end{aligned}
$$

and the Bergman kernel can be obtained by applying again the operator $\bar{D}_{z}$ from the left and $D_{w}$ from the right to each term of this expression. Correspondingly one can adapt the formulas for the orthogonal ball sectors to the case dealing with general radii $r$ and $R$.

\section{Applications to PDE}

In this section we briefly outline how the formulae that we developed can be used to treat fully analytically boundary value problems from harmonic analysis, including concrete problems from mathematical physics. The simplest examples are Dirichlet type problems in these domains. Let $\mathcal{S}_{0, \lambda, 1, k_{1}}$ be an orthogonal ball sector as introduced previously. For simplicity we use the abbreviation $\mathcal{S}$. As in [17] we introduce in these particular domains the hypercomplex Teodorescu and the Cauchy transform as

and

$$
\left(T_{\mathcal{S}}\right) f(z):=\frac{1}{A_{n+1}} \int_{\mathcal{S}} \frac{z-w}{|z-w|^{n+1}} f(w) d V(w)
$$

$$
\left(F_{\partial \mathcal{S}} u\right)(z):=\frac{1}{A_{n+1}} \int_{\partial \mathcal{S}} \frac{z-w}{|z-w|^{n+1}} n(w) u(w) d S(w)
$$

where $n(w)$ is the outer normal field at $w \in \partial \mathcal{S}$ and $z$ is an interior point of the annulus. As in [17] we denote the Bergman projection associated to the annular domain $\mathcal{S}$ by

$$
\left(P_{\mathcal{S}} f\right)(z):=\int_{\mathcal{S}} B(z, w) f(w) d V(w)
$$

where $B(z, w)$ is the Bergman kernel function which we determined for this class of domains in the previous section.

Now suppose that $f \in W_{2}^{k}\left(\mathcal{S}, C l_{0 n}\right)$ and that $g \in W_{2}^{k+3 / 2}\left(\mathcal{S}, C l_{0 n}\right)$ are arbitrarily given functions, where $W_{2}^{h}$ stands for the space of the weak-differentiable functions whose $h$-th derivatives (in the sense of Sobolev) are $L^{2}$ integrable over $\mathcal{S}$. Then, directly adapting from [17], the generalized Dirichlet problem of the form

$$
\begin{aligned}
-\Delta u(z) & =f(z) \text { in } \mathcal{S} \\
u(z) & =g(z) \text { at } \partial \mathcal{S}
\end{aligned}
$$


has a unique solution that can explicitly be represented in the closed form

$$
u=F_{\partial \mathcal{S}} g+T_{\mathcal{S}} P_{\mathcal{S}} D G+T_{\mathcal{S}} T_{\mathcal{S}} f-T_{\mathcal{S}} P_{\mathcal{S}} T_{\mathcal{S}} f \in W_{2}^{k+2, l o c}\left(\mathcal{S}, C l_{0 n}\right)
$$

where $G$ is a uniquely defined $W_{2}^{k+2}\left(\mathcal{S}, C l_{0 n}\right)$ extension of $g$. An advantage of this representation is the separation of influences on the right-hand side and the boundary data to the solution. With the explicit representation formulas for the Bergman kernel that we developed in the previous section, it is now possible to evaluate the representation formula (3) explicitly for these domains.

In the particular three-dimensional case and when $f$ is a scalar valued function, this boundary value problem models the classical heat conduction problem in annuli of a half-ball $\left(k_{1}=1\right)$ of a quarter-ball $\left(k_{1}=2\right)$ of an eighth-ball $\left(k_{1}=3\right)$ or of the full ball $\left(K_{1}=\emptyset, k_{1}:=0\right) . f$ is physically up to a multiple (the temperature conductivity) the internal heat production. $u$ represents the internal temperature and $g$ the temperature that we measure on the boundary. With the explicit knowledge of the Bergman kernel we can compute the solution $u$ fully analytically expressing it in terms of the explicit integrals given in the formula (3).

In $\mathbb{R}^{3}$ the Bergman kernel has the particular form

$$
\begin{gathered}
B(x, y)=\frac{1}{4 \pi}\left\{\sum_{k \in \mathbb{Z}}\left[D_{x} \frac{\lambda^{k}}{\left|1+\lambda^{2 k} x y\right|} D_{y}\right]\right\} \\
+\frac{1}{4 \pi}\left\{\sum _ { k \in \mathbb { Z } \emptyset \neq A \subseteq K _ { 1 } } ( - 1 ) ^ { | A | } \left[D_{x} \frac{\lambda^{k}}{\left|1+\lambda^{2 k} x y^{A}\right|} D_{y}\right.\right. \\
\left.\left.-D_{x} \frac{\lambda^{k}}{\left|y^{A}-\lambda^{2 k} x\right|} D_{y}\right]\right\},
\end{gathered}
$$

where we put $x=x_{1} e_{1}+x_{2} e_{2}+x_{3} e_{3}$ and $y=y_{1} e_{1}+y_{2} e_{2}+y_{3} e_{3}$. Notice that $\bar{y}=-y$ holds for all vectors from $\mathbb{R}^{3}$ since they have no scalar part.

Remark: This is just the simplest example among many others of partial differential equations from harmonic analysis that can be solved with these techniques and whose solutions can be expressed in terms of the Teodorescu transform, whose kernel is universal for all domains, and the Bergman projection, whose kernel is dependent on the geometry of the domain. For all these PDE (see in particular the book [17]) one can give explicit representation formulas when we have an explicit formula for the Bergman kernel. Even the solutions non linear problems like the stationary NavierStokes system with heat transfer can be expressed in terms of these integral operators, cf. also our recent paper [12]. This is just one reason why an explicit knowledge of the Bergman kernel has some importance.

\section{References}

[1] Bernstein, S. and L.S. Lanzani: Szegö projections for Hardy spaces of monogenic functions and applications. Int. Journal Math. Math. Sci. 29 (2002) No. 10, 613 - 624. 
[2] Brackx, F. and R. Delanghe: Hypercomplex function theory and Hilbert modules with reproducing kernel. Proc. London Math. Soc. 37 (1978), 545 - 576.

[3] F. Brackx, R. Delanghe and F. Sommen. Clifford Analysis. Pitman Res. Notes 76, BostonLondon-Melbourne, 1982.

[4] Brackx, F., Sommen, F. and N. Van Acker: Reproducing Bergman kernels in Clifford Analysis, Complex Variables 24 (1994), 191 - 204.

[5] Calderbank, D.: Dirac operators and Clifford analysis on manifolds with boundary. Max Planck Institute for Mathematics Bonn, Preprint number 96 - 131, 1996.

[6] Cnops, J.: Hurwitz pairs and applications of Möbius transformations. Habilitation Thesis, Ghent State University, 1993-1994.

[7] Constales, D.: The relative position of $L^{2}$ domains in complex and Clifford analysis. Ph.D. Thesis, Ghent State University, 1989-1990.

[8] Constales, D.: The Bergman and Szegö kernels for separately monogenic functions. Zeitschrift für Analysis und ihre Anwendungen 9 (1990) No. 2, 97 - 103.

[9] Constales, D. and R.S. Kraußhar: The Bergman Kernels for rectangular domains and Multiperiodic Functions in Clifford Analysis. Mathematical Methods in the Applied Sciences 25 (2002) No. 16-18, 1509 - 1526.

[10] D. Constales, R.S. Kraußhar. The Bergman Kernels for the half-ball and for fractional wedgeshaped domains in Clifford Analysis, Forum Mathematicum 17 (5) 2005, pp. 809-821.

[11] D. Constales, R.S. Kraußhar, Hilbert Spaces of Solutions to Polynomial Dirac equations, Fourier Transforms and Reproducing Kernel Functions for Cylindrical Domains, Zeitschrift für Analysis und Ihre Anwendungen 24 (3) 2005, pp. 611-636.

[12] D. Constales, R.S. Kraußhar, On the Navier-Stokes equation with Free Convection in three dimensional triangular channels, Mathematical Methods in the Applied Sciences 316 (2008), pp. 735-751.

[13] D. Constales, D. Grob and R.S. Kraußhar, Constructing 3D mappings onto the unit sphere with the hypercomplex Szegö kernel, to appear in J. Comput. Appl.Math.

[14] Delanghe, R.: On Hilbert modules with reproducing kernel. Funct. theor. Meth. part. Differ. Equat., Proc. int. Symp., Darmstadt 1976, Lect. Notes Math. 561 (1976), 158 - 170.

[15] Delanghe, R., Sommen, F. and V. Souček: Clifford Algebra and Spinor Valued Functions, Dordrecht-Boston-London: Kluwer 1992.

[16] Eastwood, M. and J. Ryan: Aspects of Dirac operators in analysis. Milan J. Math. 75 (2007), 91-116.

[17] Gürlebeck, K. and W. Sprössig: Quaternionic analysis and elliptic boundary value problems, Basel: Birkhäuser, 1990.

[18] Kravchenko, V. and M. Shapiro: Helmholtz operator with a quaternionic wave number and associated function theory. In: Ławrynowicz, J. (ed.), Deformations of mathematical structures 2: Hurwitz-type structures and applications to surface physics. Selected papers from the seminar on deformations, Lódz-Malinka, Poland, 1988-1992, Dordrecht: Kluwer 1994, 101 - 128.

[19] Kravchenko, V. and M. Shapiro: Integral representations for spatial models of mathematical physics, Harlow: Addison Wesley Longman 1996.

[20] Ryan, J.: Hilbert modules with reproducing kernels within complex Clifford analysis. Mathematical structure - computational mathematics - mathematical modelling 2, Sofia: Bulgar. Acad. Sci. 1984, $273-278$. 
[21] Shapiro, M. and N. Vasilevski: On the Bergman kernel function in the Clifford analysis. In: Clifford algebras and their applications in mathematical physics, Fund. Theor. Phys. 55, Dortrecht: Kluwer 1993, 183 - 192.

[22] Shapiro, M. and N. Vasilevski: On the Bergmann kernel function in hyperholomorphic analysis. Acta Appl. Math. 46 (1997) No. 1, 1- 27.

Denis Constales

Department of Mathematical Analysis, Ghent University, Building S-22, Galglaan 2, B-9000 Ghent, Belgium.

e-mail: dc@cage. UGent. be

Dennis Grob

Lehrstuhl A für Mathematik, Rheinisch-Westfälische Technische Hochschule Aachen, D-52056 Aachen, Germany.

e-mail: dennis.grob@mathA.rwth-aachen. de

Rolf Sören Kraußhar

Institut für Mathematik, Universität Paderborn, Warburgerstr.100, 33098 Paderborn, Germany. e-mail: soeren.krausshareuni-paderborn.de 